\title{
ESTIMATIVA DA VELOCIDADE DE INFILTRAÇÃO BÁSICA DO SOLO'
}

\author{
LUÍS EDUARDO DE OLIVEIRA SALES ${ }^{2}$, MOZART MARTINS FERREIRA ${ }^{3}$, \\ MARCELO SILVA DE OLIVEIRA ${ }^{4}$ e NILTON CURI ${ }^{3}$
}

\begin{abstract}
RESUMO - Objetivando estimar a velocidade de infiltração básica (VIB), avaliou-se a associação desta com outras propriedades físicas das camadas superficial e subsuperficial de um Latossolo Roxo e um Podzólico Vermelho-Amarelo, ambos ocorrentes no campus da Universidade Federal de Lavras. Para tanto, delineou-se um plano amostral consistindo de uma rede cujas malhas apresentaram espaçamentos variáveis. Nos pontos de interseção das malhas determinou-se a VIB e, posteriormente, coletaram-se amostras com estrutura deformada e indeformada nas camadas de 0-20 e 60-80 cm. Com essas amostras determinou-se a textura, densidade do solo e de partículas, porosidade total, macro e microporosidade e condutividade hidráulica do solo saturado. Após análises estatísticas de correlação e regressão, verificou-se que as propriedades físicas da camada de 0-20 cm do Latossolo Roxo e 60-80 cm do Podzólico Vermelho-Amarelo associaram-se melhor com a VIB. Tanto a condutividade hidráulica do solo saturado quanto a densidade do solo se mostraram adequadas para estimar a VIB desses solos.
\end{abstract}

Termos para indexação: Latossolo Roxo, Podzólico Vermelho-Amarelo, condutividade hidráulica do solo saturado, densidade do solo.

\section{ESTIMATION OF THE SOIL BASIC INFILTRATION VELOCITY}

ABSTRACT - With the objective of estimating the soil basic infiltration velocity (BIV), a study was conducted to evaluate the degree of association between BIV and other physical properties of the superficial and subsuperficial layers of a Dusky-Red Latosol (Oxisol) and of a Red-Yellow Podzolic soil (Ultisol), both located at the campus of the Universidade Federal de Lavras, Minas Gerais State, Brazil. The sampling was performed with systematic spacing and the points constituted a grid, with variable spacing. At each sampling point, the value of the basic infiltration velocity was determined and thereafter undisturbed and disturbed samples at $0-20$ and $60-80 \mathrm{~cm}$ of depth were collected. With these samples, the particle-size distribution, bulk density, particle density, total volume of pores, macroporosity, microporosity and hydraulic conductivity of saturated soil were determined. Statistical analysis of correlation and regression showed that the physical properties of the $0-20 \mathrm{~cm}$ layer in the Dusky-Red Latosol and of the 60-80 cm layer in the Red-Yellow Podzolic soil were better associated with the BIV. Both hydraulic conductivity of saturated soil and bulk density were adequate for estimating the BIV of these soils.

Index terms: Dusky-Red Latosol, Red-Yellow Podzolic soil, hydraulic conductivity of saturated soil, bulk density.

\section{INTRODUÇ̃̃O}

A capacidade de infiltração é uma propriedade do solo que representa a intensidade máxima que o solo,

\footnotetext{
${ }^{1}$ Aceito para publicação em 7 de abril de 1999.

Extraído da Dissertação de Mestrado apresentada pelo primeiro autor à Universidade Federal de Lavras (UFLA).

${ }^{2}$ Eng. Agríc., M.Sc., FIMES, Caixa Postal 104, CEP 75830-000 Mineiros, GO

${ }^{3}$ Eng. Agr., Ph.D., Dep. de Ciência do Solo, UFLA, Caixa Postal 37, CEP 37200-000 Lavras, MG. E-mail: mozartmf@ufla.br

${ }^{4}$ Eng. Agr., Ph.D., Dep. de Ciências Exatas, UFLA. E-mail: marcelso@ufla.br
}

em dada condição e tempo, pode absorver a água da chuva ou da irrigação aplicada a determinada taxa. Esse processo, contudo, não é constante ao longo do tempo. Por ocasião de uma chuva ou irrigação, a velocidade de infiltração é máxima no início do evento, e decresce rapidamente, de acordo com as condições do solo. Sob chuva ou irrigação contínua, a velocidade de infiltração se aproxima, gradualmente, de um valor mínimo e constante. Esse valor constante que a velocidade de infiltração atinge com o passar do tempo é conhecido por velocidade de infiltração básica (Bernardo, 1989).

De acordo com Araújo Filho \& Ribeiro (1996), na elaboração de projetos de irrigação, a magnitude da 
velocidade de infiltração básica serve de referência para a escolha dos métodos e sistemas de irrigação a adotar, sendo, portanto, uma variável relevante para a definição do manejo agrícola em sistemas irrigados.

Vários são os fatores do solo que interferem na magnitude da velocidade de infiltração básica. Esses fatores estão associados às propriedades físicas do solo, da própria água, e do manejo adotado. Textura (Daker, 1970), estrutura (Resende et al., 1997), tamanho e disposição do espaço poroso (Baver et al., 1972), manejo do solo (Dickerson, 1976; Machado, 1976), mineralogia (Ferreira, 1988) e umidade inicial, para solos com argilas expansivas (Araújo Filho \& Ribeiro, 1996; Jabro, 1996), além da metodologia utilizada (Brito et al., 1996), são alguns dos fatores apontados como responsáveis pelas variações nos valores da velocidade de infiltração básica dos solos.

A recomendação corrente é que a determinação da velocidade de infiltração básica seja executada in situ, de forma mais representativa e precisa possível. Contudo, dada a grande variabilidade espacial a que os solos estão submetidos, a observância dessa recomendação tem tornado aquela determinação uma tarefa das mais laboriosas e cujos resultados se acham envoltos por muitos questionamentos. Em razão desses aspectos, Libardi (1995) comenta que muitos pesquisadores têm procurado calculá-la teoricamente, a partir de outras propriedades do solo de mais fácil medida. Estudos nessa direção foram desenvolvidos por Ahuja et al. (1984) e Miyazaki (1996), utilizando respectivamente a macroporosidade e a densidade do solo.

Dada a carência de informações envolvendo solos tropicais, delineou-se o presente estudo, cujo objetivo é estimar a velocidade de infiltração básica a partir de outros atributos físicos do solo a ela associados, mas de mensuração mais fácil e de maior reprodutibilidade.

\section{MATERIAL E MÉTODOS}

O trabalho foi conduzido em dois solos ocorrentes no campus da Universidade Federal de Lavras: um Latossolo Roxo, textura muito argilosa, estrutura granular, e um Podzólico Vermelho-Amarelo, textura argilosa, estrutura em blocos. O plano amostral utilizado consistiu de amostragem sistemática com espaçamentos irregulares. Os pontos amostrais consistiram das interseções de uma malha quadriculada (grid), com espaçamentos variáveis de $0,56 \mathrm{~m}$ até $15 \mathrm{~m}$, totalizando 40 pontos amostrais.

Em cada ponto amostral das áreas determinou-se a velocidade de infiltração básica (VIB), utilizando-se o infiltrômetro de anéis concêntricos, de acordo com Bernardo (1989). Após as determinações da VIB, coletaram-se amostras de solo nas camadas de $0-20$ e $60-80 \mathrm{~cm}$, representativas dos horizontes diagnósticos de superfície e subsuperfície respectivamente, com estrutura indeformada e deformada. As amostras com estrutura indeformada foram submetidas às seguintes determinações: densidade do solo (Ds), determinada pelo método do anel volumétrico (Blake, 1965a); microporosidade (micro), determinada pela unidade de sucção, segundo Grohmann (1960); porosidade total (VTP), calculada segundo proposição de Vomocil (1965); macroporosidade (macro), calculada pela diferença entre a porosidade total e a microporosidade; e condutividade hidráulica do solo saturado (Ks), determinada pelo permeâmetro de carga constante, segundo Klute (1965). As amostras com estrutura deformada foram submetidas às seguintes determinações: densidade de partículas (Dp), pelo picnômetro e água destilada desaerada a vácuo, conforme Blake (1965b), e análise granulométrica, pelo método de Bouyoucos modificado (Carvalho, 1985). Para quantificar a associação entre a velocidade de infiltração básica e demais propriedades físicas avaliadas, em ambos os solos e camadas, foram submetidos os resultados das avaliações às análises estatísticas de correlação e regressão. Para tanto, utilizou-se o Sistema para Análises Estatísticas (SAEG), da Universidade Federal de Viçosa.

\section{RESULTADOS E DISCUSSÃO}

Os resultados das determinações da Velocidade de Infiltração Básica (VIB) e das propriedades físicas das camadas de 0-20 cm e 60-80 cm do Podzólico Vermelho-Amarelo e Latossolo Roxo são apresentados na Tabela 1. Observa-se que os valores da VIB são bastante contrastantes: $12,1 \mathrm{~mm} \mathrm{~h}^{-1}$ no Podzólico Vermelho-Amarelo, e 56,6 $\mathrm{mm} \mathrm{h}^{-1}$ no Latossolo Roxo. Em princípio, pode-se associar esses resultados às distintas características morfológicas associadas à estrutura dos horizontes desses solos, que foram avaliadas nos estudos desenvolvidos por Andrade (1976) e Rocha (1982).

Ao se comparar os comportamentos das propriedades físicas obtidas a partir das amostras coletadas 
nas camadas de 0-20 cm e $60-80 \mathrm{~cm}$, verifica-se que as tendências nos dois solos são bastante diferentes. A condutividade hidráulica do solo saturado $(\mathrm{Ks})$ passa de $41,30 \mathrm{~mm} \mathrm{~h}^{-1}$ na camada de $0-20$ para $15,16 \mathrm{~mm} \mathrm{~h}^{-1}$ na camada de $60-80 \mathrm{~cm}$ no Podzólico Vermelho-Amarelo, e de $21,18 \mathrm{~mm} \mathrm{~h}^{-1}$ para $81,88 \mathrm{~mm} \mathrm{~h}^{-1}$ nas mesmas camadas no Latossolo Roxo. Em ambos os solos, os valores de Ds são maiores na camada superficial, não havendo, contudo, diferenças entre os valores de Dp entre as camadas dentro de cada solo. Os valores encontrados para Volume Total de Poros (VTP) dos dois solos seguem a mesma tendência da Ds e são mais elevados no Latossolo Roxo. Com relação à distribuição de poros por tamanho (macro e microporosidade), observa-se, inicialmente, que o Latossolo Roxo, embora bem mais argiloso, apresenta maior macroporosidade. A concentração de microporos nos dois solos é bem semelhante.

Com o intuito de avaliar o grau de associação entre a VIB e essas propriedades físicas dos solos, visando a sua estimativa, foram efetuados estudos de correlação cujos resultados encontram-se na Tabela 2.

Observa-se, na Tabela 2, que a VIB correlacionou-se significativamente, em ambos os solos e camadas, com Ks, Ds, VTP e Macroporosidade, sendo inversa apenas a correlação entre VIB e Ds. Entretanto, os maiores coeficientes das correlações foram encontrados na camada de $60-80 \mathrm{~cm}$ do Podzólico Vermelho-Amarelo e na camada de $0-20 \mathrm{~cm}$ do Latossolo Roxo. Esses resultados confirmam as exposições apresentadas anteriormente por Daker (1970), Baver et al. (1972) e Resende et al. (1997). Considerando os resultados das análises de correlações e as peculiaridades que envolvem cada propriedade física determinada, é razoável admitir que as melhores estimativas da VIB seriam obtidas a partir de Ks e Ds. Resultado semelhante, envolvendo a Ds, foi encontrado por Miyazaki (1996).

Tomando por base o estudo de correlação, foram estabelecidas relações funcionais envolvendo a VIB,

TABELA 1. Velocidade de infiltração básica (VIB) e demais propriedades dos solos ${ }^{1}$.

\begin{tabular}{|c|c|c|c|c|c|c|c|c|c|c|}
\hline $\begin{array}{c}\text { Profundidade } \\
(\mathrm{cm})\end{array}$ & $\begin{array}{l}\text { VIB } \\
----(\mathrm{mn}\end{array}$ & $\begin{array}{c}\mathrm{Ks} \\
\left.\mathrm{h}^{-1}\right)---\end{array}$ & $\begin{array}{l}\text { Ds } \\
---(\mathrm{g} \mathrm{cm}\end{array}$ & $\begin{array}{c}\mathrm{Dp} \\
-3)--- \\
\end{array}$ & $\begin{array}{l}\text { VTP } \\
----- \\
\end{array}$ & $\begin{array}{l}\text { Macro } \\
--(\%)\end{array}$ & Micro & Argila & $\begin{array}{l}\text { Silte } \\
\left(\mathrm{g} \mathrm{kg}^{-1}\right)\end{array}$ & Areia \\
\hline & \multicolumn{10}{|c|}{ Podzólico Vermelho-Amarelo } \\
\hline $0-20$ & 12,1 & 41,30 & 1,40 & 2,61 & 46,50 & 7,18 & 39,32 & 422 & 155 & 423 \\
\hline $60-80$ & - & 15,16 & 1,35 & 2,65 & 49,03 & 5,18 & 43,86 & 524 & 112 & 364 \\
\hline & \multicolumn{10}{|c|}{ Latossolo Roxo } \\
\hline $0-20$ & 56,6 & 21,18 & 1,19 & 2,82 & 58,24 & 16,82 & 41,42 & 653 & 152 & 195 \\
\hline $60-80$ & - & 81,88 & 0,97 & 2,85 & 65,48 & 31,35 & 34,23 & 725 & 136 & 139 \\
\hline
\end{tabular}

${ }^{1}$ Ks: condutividade hidráulica do solo saturado; Ds: densidade do solo; Dp: densidade de partículas; VTP: volume total de poros; Macro: macroporosidade; Micro: microporosidade.

TABELA2. Coeficientes das correlações entre a velocidade de infiltração básica (VIB) e condutividade hidráulica do solo saturado (Ks), densidade do solo (Ds), volume total de poros (VTP) e macroporosidade (Macro) das camadas de 0-20 e 60-80 cm dos solos.

\begin{tabular}{|c|c|c|c|c|}
\hline Profundidade $(\mathrm{cm})$ & $\mathrm{Ks}\left(\mathrm{mm} \mathrm{h}^{-1}\right)$ & Ds $\left(\mathrm{g} \mathrm{cm}^{-3}\right)$ & $\operatorname{VTP}(\%)$ & Macro (\%) \\
\hline & \multicolumn{4}{|c|}{ Podzólico Vermelho-Amarelo } \\
\hline $0-20$ & $0,95^{*}$ & $-0,87^{*}$ & $0,86^{*}$ & $0,91 *$ \\
\hline \multirow[t]{2}{*}{$60-80$} & $0,97 *$ & $-0,96^{*}$ & $0,96 *$ & $0,92 *$ \\
\hline & \multicolumn{4}{|c|}{ Latossolo Roxo } \\
\hline $0-20$ & $0,80^{*}$ & $-0,76^{*}$ & $0,75^{*}$ & $0,70 *$ \\
\hline $60-80$ & $0,79 *$ & $-0,69^{*}$ & $0,69 *$ & $0,69 *$ \\
\hline
\end{tabular}


Ks e Ds, cujos resultados são apresentados nas Figs. 1 e 2 (Latossolo Roxo) e Figs. 3 e 4 (Podzólico Vermelho-Amarelo). Observa-se, nessas figuras, que os modelos para previsão da VIB são similares nos

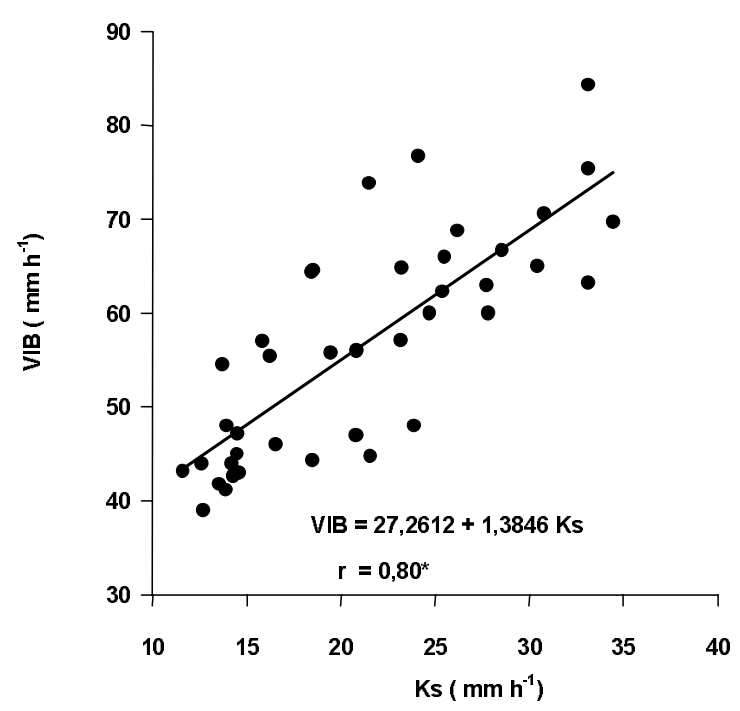

FIG. 1. Relação entre a velocidade de infiltração básica (VIB) e a condutividade hidráulica do solo saturado (Ks) da camada de $0-20 \mathrm{~cm}$ do Latossolo Roxo.

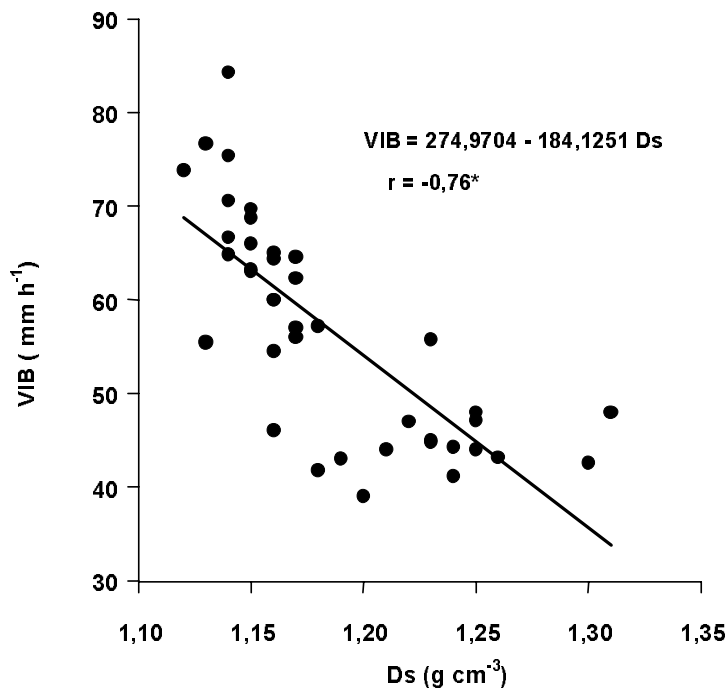

FIG. 2. Relação entre a velocidade de infiltração básica (VIB) e a densidade do solo (Ds) da camada de 0-20 cm do Latossolo Roxo. dois solos. Contudo, os melhores ajustes foram obtidos no que tange ao Podzólico Vermelho-Amarelo, refletindo menor variabilidade espacial deste em relação ao Latossolo Roxo.

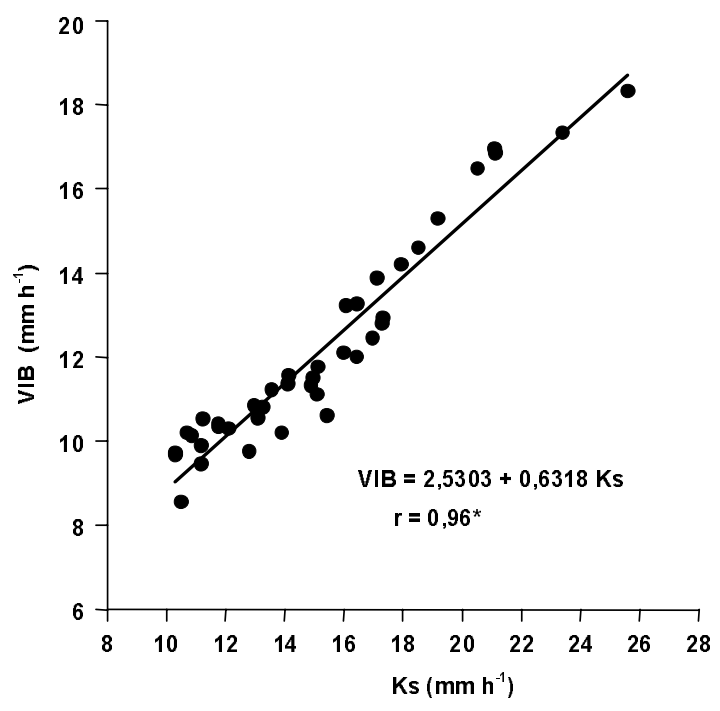

FIG. 3. Relação entre a velocidade de infiltração básica (VIB) e a condutividade hidráulica do solo saturado (Ks) da camada de $60-80 \mathrm{~cm}$ do Podzólico Vermelho-Amarelo.

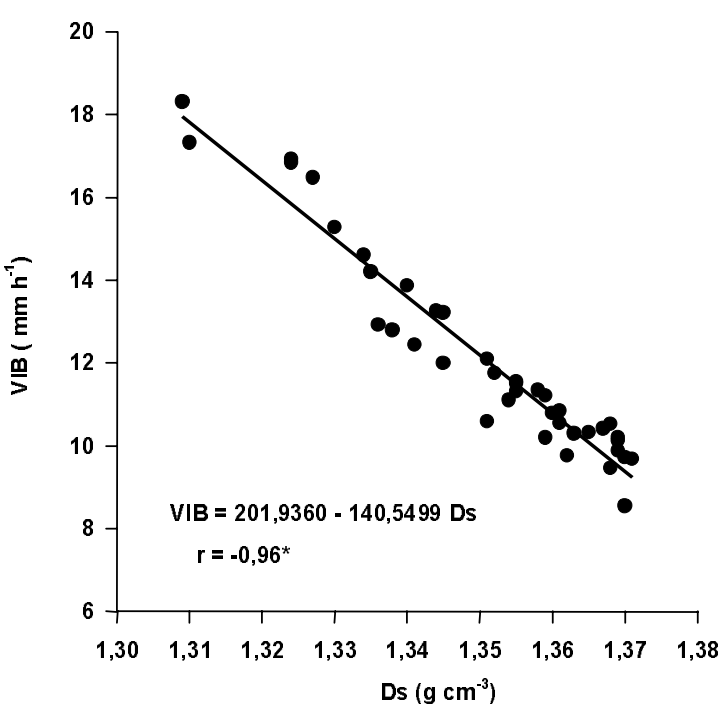

FIG. 4. Relação entre a velocidade de infilttração básica (VIB) e a densidade do solo (Ds) da camada de 60-80 cm do Podzólico VermelhoAmarelo. 


\section{CONCLUSÕES}

1. As propriedades físicas da camada de $60-80 \mathrm{~cm}$ do Podzólico Vermelho-Amarelo e $0-20 \mathrm{~cm}$ do Latossolo Roxo se associam melhor com a velocidade de infiltração básica.

2. A condutividade hidráulica do solo saturado e a densidade do solo se mostram adequadas para se estimar a velocidade de infiltração básica dos solos.

\section{REFERÊNCIAS}

AHUJA, L.R.; NANEY, J.W.; GREEN, R.E.; NIELSEN, D.R. Macroporosity to characterize spatial variability of hydraulic conductivity and effects of land management. Soil Science Society of America. Journal, Madison, v.48, p.699-702, 1984.

ANDRADE, H. Caracterização genética, morfológica e classificação de dois solos do município de Lavras-MG, em correlação com a geomorfologia da área. Lavras: ESAL, 1976. 76p. Tese de Mestrado

ARAÚJO FILHO, J.C. de; RIBEIRO, M.R. Infilltração de água em Cambissolos do Baixio de Irecê (BA). Revista Brasileira de Ciência do Solo, Campinas, v.20, n.3, p.363-370, 1996

BAVER, L.D.; GARDNER, W.H.; GARDNER, W.R. Soil physics. 4.ed. New York: John Wiley, 1972. 498p.

BERNARDO, S. Manual de irrigação. 5.ed. Viçosa UFV, Imprensa Universitária, 1989. 596p.

BLAKE, G.R. Bulk density. In: BLACK, C.A (Ed.) Methods of soil analysis: physical and mineralogical properties, including statistics of measurement and sampling. Madison: American Society of Agronomy, 1965a. pt.1, p.374-390

BLAKE, G.R. Particle density. In: BLACK, C.A. (Ed.) Methods of soil analysis: physical and mineralogical properties, including statistics of measurement and sampling. Madison: American Society of Agronomy, 1965b. pt.1, p.371-373

BRITO, L.T. de L.; LOUREIRO, B.T.; DENICULI, W.; RAMOS, M.M.; SOARES, J.M. Influência do método na determinação da velocidade de infiltração. Revista Brasileira de Ciência do Solo, Campinas, v.20, n.3, p.503-507, 1996.

CARVALHO, M.A. de. Eficiência de dispersantes na análise textural de materiais de solos com hori- zontes B latossólico e B textural. Lavras: ESAL, 1985. 79p. Tese de Mestrado.

DAKER, A. A água na agricultura: irrigação e drenagem. 3.ed. Rio de Janeiro: Freitas Bastos, 1970. v.3, $453 \mathrm{p}$

DICKERSON, B.P. Soil compactation after tree-length skidding in northern Mississipi. Soil Science Society of America. Journal, Madison, v.40, n.6, p.965-966, Nov./Dec. 1976

FERREIRA, M.M. Influência da mineralogia da fração argila nas propriedades físicas de latossolos brasileiros. Viçosa: UFV, 1988. 79p. Tese de Doutorado.

GROHMANN, F. Distribuição do tamanho de poros de três tipos de solos do Estado de São Paulo. Bragantia, Campinas, v.19, n.21, p.319-328, abr 1960

JABRO, J.D. Variability of field-saturated hydraulic conductivity in hagerstown soil as affected by initial water content. Soil Science, Baltimore, v.161, n.11, p.735-739, 1996.

KLUTE, A. Laboratory measurement of hydraulic conductivity of saturated soil. In: BLACK, C.A (Ed.). Methods of soil analysis: physical and mineralogical properties, including statistics of measurement and sampling. Madison: American Society of Agronomy, 1965. pt.1, p.253-261.

LIBARDI, P.L. (Ed.). Dinâmica da água no solo Piracicaba: ESALQ-USP, 1995. 497p.

MACHADO, J.A. Efeito do sistema convencional de cultivo na capacidade de infiltração da água no solo. Santa Maria, RS: CCR, UFSM, 1976. 133p. Tese de Mestrado

MIYAZAKI, T. Bulk density dependence of air entry suctions and saturated hydraulic conductivities of soils. Soil Science, Baltimore, v. 161, n.8, p.484-490, Aug. 1996.

RESENDE, M.; CURI, N.; REZENDE, S.B.; CORRÊA, G.F. Pedologia: base para distinção de ambientes. 2.ed. Viçosa: NEPUT, 1997. 367p.

ROCHA, G.C. Geologia, geomorfologia e pedologia de uma catena de solos situada no Campus da Escola Superior de Agricultura de Lavras, MG. Lavras: ESAL, 1982. 109p. Tese de Mestrado.

VOMOCIL, J.A. Porosity. In: BLACK, C.A. (Ed.) Methods of soil analysis: physical and mineralogical properties, including statistics of measurement and sampling. Madison: American Society of Agronomy, 1965. pt.1, p.499-510. 